\title{
London hospital launches infectious disease 'biobank'
}

British doctors have begun collecting samples for Britain's first storehouse of infectious diseases, two years after the launch of the UK's ambitious BioBank project.

Following nearly a year of discussion over ethical approval, Guy's \& St Thomas' hospital in London has a $\mathfrak{E} 45$ million share of a $\mathfrak{E} 450$ million government initiative for developing 11 biomedical research centres across England. The hospital's $\mathfrak{E} 1$ million infectious disease biobank is intended to offer researchers a resource for uncovering which genes render some people more susceptible to infectious diseases than others.

Over the next three years, the scientists hope to collect 3,000 samples from those infected with HIV, hepatitis $B$ virus and

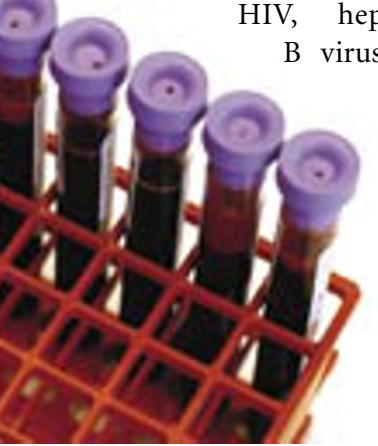

multidrug-resistant Staphylococcus aureus. Blood and plasma will be drawn from specific target groups and stored for up to 15 years.

Researchers are already recruiting 700 people from a south London area, which they say has a diverse ethnic makeup. For instance, most HIV-infected people in the UK and the US carry a subtype of the HIV virus dubbed clade B. In Africa, India and Southeast Asia, however, clade $\mathrm{C}$ is more prevalent.

This south London area "is one of the few places which have patients infected with diverse clades," says Anna Vyakarnum, the biobank's primary researcher and a senior lecturer in infectious disease at Kings College London.

Britain also hosts the world's biggest biobank, which aims to collect 15 million samples to probe the effect on health of the interaction between genes and the environment.

Critics have warned that these biomedical repositories pose serious ethical concerns. (Nat. Med. 11, 696; 2005) Without proper safeguards, pharmaceutical companies could gain access to the samples and then patent the gene sequences linked to a disease-in essence monopolizing the testing and treatments for certain infections, notes Helen Wallace, deputy director of Genewatch UK, a Derbyshire-based watchdog group.

In June 2006, a senior employee of the US
National Institutes of Health pleaded guilty to shipping samples of human spinal fluid to Pfizer without approval from his employers (Nature 441, 912-913; 2006).

"The problem with this is that you end up with lots of different companies owning different pieces of the jigsaw," says Wallace. "Any treatment that needs to reach poor people then involves complex license negotiations." The Kings handout on the project doesn't clearly warn participants about these potential outcomes, she adds.

Another concern is that commercial interests may set priority on what type of research is undertaken. The Kings biobank intends to charge a preparation fee of around $\mathfrak{£} 150$ for samples. "The problem with this is it does make the use of that tissue fairly exclusive, smaller academic groups may not be able to afford it and be left out," says David Hunter, a lecturer in bioethics at the University of Ulster in Coleraine.

John Cason, head of the infectious disease biobank and senior lecturer in virology at Kings College, says samples will not be sold.

"We would ask for a contribution towards the processing costs to just cover our working costs," Cason explains. "These get cheaper the more samples we get."

Narelle Towie, London

On 27 April, the US Food and Drug Administration rejected Merck's application for Arcoxia, a Cox-2-targeting painkiller widely seen as the successor to the ill-fated Vioxx. The fate of Vioxx, Arcoxia's rejection-and the dearth of other effective painkillers in the market-begs the question...

\section{Is it possible to make a Cox-2 inhibitor that's safe?}

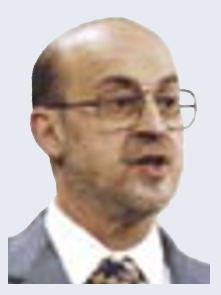

Steve Nissen

Chairman, Department of

Cardiovascular Medicine

Cleveland Clinic

Foundation

We still don't know if all

Cox-2 inhibitors carry

a higher risk than all conventional nonsteroidal anti-inflammatory drugs, so the issue of relative risk is still an open question.

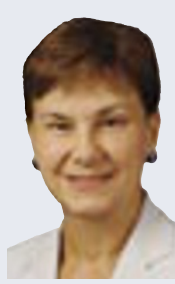

Janet Woodcock

Deputy commissioner and

chief medical officer

US Food and Drug

Administration

No pharmacologically active agent is 'safe'. A molecule with

some Cox-2 inhibitory action might be engineered to have less cardiovascular toxicity but might still have renal, gastrointestinal or other serious toxicity.

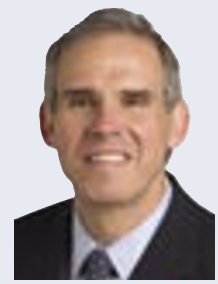

Eric Topol

Director Scripps Translational Science Institute It hasn't been done yet. But if we could figure out the mechanism of cardiovascular toxicity, I wouldn't rule it out as impossible.

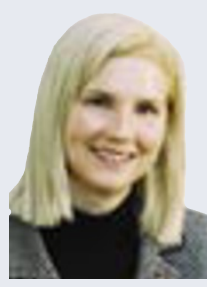

Donna Arnett

Chair, Department of Epidemiology

University of Alabama at Birmingham

Given the consistent and well documented excess cardiovascular risk associated with Cox-2 inhibitors, and the availability of other anti-inflammatory drugs that do not cause cardiovascular toxicity, what is the motivation for trying to make a new one?

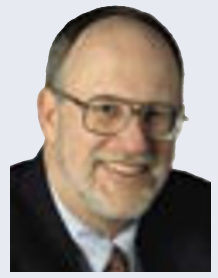

Bruce Psaty

Professor of medicine

University of Washington Cardiovascular Health Research Unit

It's difficult to predict, but a large randomized clinical trial will need to demonstrate that, compared with the best available treatment, the benefits of the new drug outweigh the risks. 